\title{
The X-ray spectrum of the black hole candidate GX339-4 in a low state
}

\author{
A. Corongiu ${ }^{1-3}$, L. Chiappetti ${ }^{1}$, F. Haardt ${ }^{4}$, A. Treves ${ }^{4}$, M. Colpi $^{5}$, and T. Belloni ${ }^{6}$ \\ 1 IASF-CNR, Sezione di Milano “G. Occhialini”, via Bassini 15, 20133 Milano, Italy \\ 2 Dipartimento di Scienze Fisiche, Università degli Studi di Milano, via Celoria 16, 20133 Milano, Italy \\ 3 INAF - Osservatorio Astronomico di Cagliari, Loc. Poggio dei Pini, strada 54, 09012 Capoterra, Italy \\ ${ }^{4}$ Dipartimento di Scienze, Università dell'Insubria/Como, via Valleggio 11, 22100 Como, Italy \\ 5 Dipartimento di Fisica, Università di Milano Bicocca, piazza delle Scienze 3, 20126 Milano, Italy \\ ${ }^{6}$ INAF - Osservatorio Astronomico di Brera, via E. Bianchi 46, 23807 Merate, Italy
}

Received 22 April 2003 / Accepted 12 June 2003

\begin{abstract}
We report on four observations of the black hole candidate GX 339-4 collected in the spectral range 0.4-140 keV with the BeppoSAX satellite during 1997. All our spectra are typical of a black hole candidate in its low-hard state, i.e. are dominated by the hard tail of the emission from the corona. Although the direct emission from the accretion disk in the soft $\mathrm{X}$-ray range is weak, spectra are well described only with models that take into consideration the Compton reflection of hard $\mathrm{X}$-rays from the corona onto the surface of an accretion disk of non negligible extent.
\end{abstract}

Key words. accretion, accretion disks - binaries: general - stars: individual (GX 339-4) - X-rays: stars - black hole physics

\section{Introduction}

GX 339-4, discovered in 1971 by Markert et al. (1973a, 1973b), was proposed to be a black hole candidate by Samimi et al. (1979), because of its spectral features and its short term variability similar to sources like Cyg X-1.

GX 339-4 was observed in five different spectral states, each one with well defined features (Tanaka \& Lewin 1995). Most observations detected the source in the hard-low state, and often with such a low count rate to properly call it an off state; less often the source was detected in the high-soft state, only twice in the very high state (before 2001) and twice in the intermediate state (for a recent re-consideration of the nature of the intermediate state see Homan et al. 2001). In the first half of 1999, the source entered an extended off-state, and it remained undetectable by the All-Sky Monitor on board RossiXTE (see Kong et al. 2000; Corbel et al. 2003). During 2002 GX 339-4 started a new outburst (Smith et al. 2002) with more state transitions (Belloni et al. 2003) The light curve from the RXTE/ASM is shown in Fig. 1.

In its low state GX 339-4 shows power law spectra, with photon index $\Gamma=1.62-2.30$ (Dolan et al. 1987; Bouchet et al. 1993; Ueda et al. 1994; Mendez \& van der Klis 1997; Zdziarski et al. 1998; Belloni et al. 1999; Wilms et al. 1999). Higher values for $\Gamma$ were detected in spectra observed up to $200 \mathrm{keV}(\Gamma=2.0 \pm 0.3$; Dolan et al. 1987) and to $1.3 \mathrm{MeV}$

Send offprint requests to: A. Corongiu, e-mail: corongiu@ca.astro.it
(Bouchet et al. 1993). Most observations in the low state indicate the presence of the fluorescence Fe line (Ueda et al. 1994; Zdziarski et al. 1998; Dolan et al. 1987), with an equivalent width of about $50-100 \mathrm{eV}$.

Several authors (Zdziarski et al. 1998; Wilms et al. 1999; Revnivtsev et al. 2001; Nowak et al. 2002; Wardziński et al. 2002) give indication of a reflection component in the $X$ spectra, described according to the model of Magdziarz \& Zdziarski (1995) and provide evidence for the Fe abundance being greater by a factor $2-3$ relative to solar abundance.

GX 339-4 high states have also well defined spectral features. In this state the spectrum shows a soft component, due to the thermal emission by an accretion disk (Makishima et al. 1986; Belloni et al. 1999), described by a multicolour blackbody model (Makishima et al. 1986). Inner temperatures of the disk were estimated to be $0.77 \mathrm{keV}$ (Makishima et al. 1986) and $0.63-0.72 \mathrm{keV}$ (Belloni et al. 1999). The high energy part of the observed spectra has a powerlaw behaviour, with a photon index $\Gamma$ of 2.0 (Makishima et al. 1986) and of 2.12-2.57 (Belloni et al. 1999).

In the high state the fluorescence $\mathrm{Fe}$ line at $6.4 \mathrm{keV}$ is present, with an equivalent width of $319-350 \mathrm{eV}$. There is also evidence for an absorption edge, although its central energy is uncertain: Makishima et al. (1986) report a value of $8.8 \mathrm{keV}$, while Belloni et al. (1999) one of $6.69 \mathrm{keV}$.

The source is very active also in the radio band, with radio flux strictly correlated to the X-ray intensity (Corbel et al. 2003; Markoff et al. 2003). 


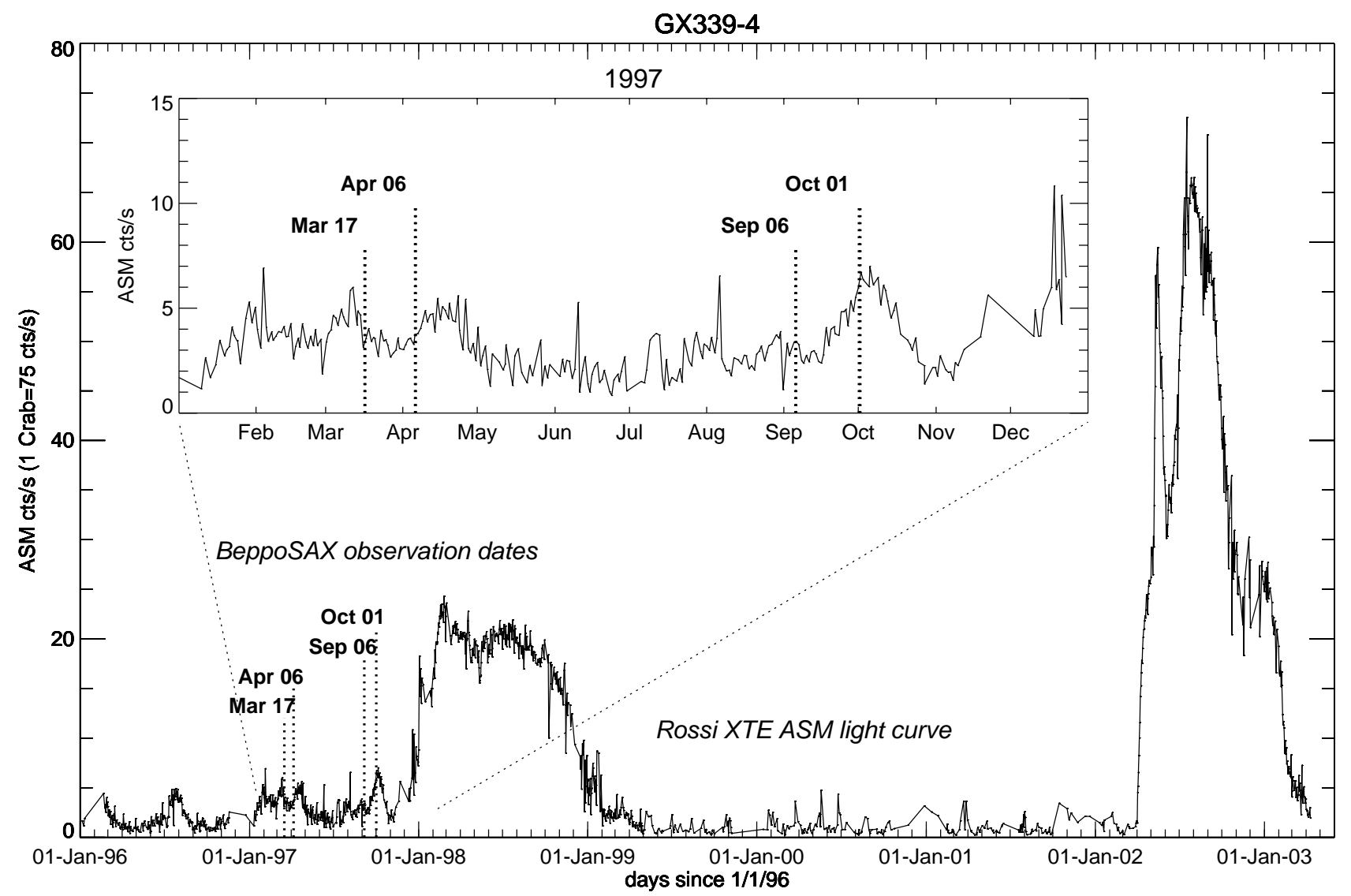

Fig. 1. Historical GX 339-4 light curve as observed by the All Sky Monitor onboard Rossi XTE. The dashed vertical lines in the main panel and in the inset indicate the epoch of our BeppoSAX observations.

In this paper we report results from four observations of GX 339-4, taken during 1997 with the BeppoSAX satellite, when the source was in a low-hard state. Using all four narrow field instruments available in the satellite scientific payload, we obtained and analysed data in the $0.4-140.0 \mathrm{keV}$ band. In Sect. 2 we present the four observations with a summary of the reduction procedure; in Sect. 3 we describe the data analysis and in Sect. 4 we discuss our results.

\section{Observations and data reduction}

We observed GX 339-4 with BeppoSAX (Boella et al. 1997 and references therein) at four epochs during 1997, with the primary aim to study the spectral variability on the 1-month and 6-month time scale. A journal with basic data is reported in Table 1, while the state of the source at the epoch of our observations with respect to the historical light curve is visible in Fig. 1. Preliminary results on the first two observations have been previously reported by Chiappetti et al. (1998).

From the figure it is apparent that we observed the source at only slightly different intensity levels during a low state.

We detected GX 339-4 with all the four Narrow Field Instruments on board BeppoSAX, obtaining a simultaneous coverage with an excellent signal to noise from 0.4 to $140 \mathrm{keV}$. MECS (Medium Energy Concentrator Spectrometer) observations used all three MECS units for the first two observations, and only two units for the last two, due to a failure of unit MECS1 in late spring 1997.

Data reduction for MECS, HPGSPC (High Pressure Gas Scintillation Proportional Counter) and PDS (Phoswich Detector System) was performed using the XAS software (Chiappetti \& Dal Fiume 1997), while for LECS (Low Energy Concentrator Spectrometer) data we used the cleaned event files supplied by the ASI Science Data Centre (ASDC). In general the reduction followed the standard prescriptions described e.g. in Chiappetti et al. (1999), with only slight differences and peculiarities due to the higher intensity of GX 339-4. A summary is given below. The reader not interested in reduction details may wish to proceed directly to Sect. 3 .

We examined the light curves of GX 339-4 during our (relatively short) observations, but, since we found no indication of large variability, we proceeded accumulating a single spectrum per instrument for each observation.

LECS gross spectra were extracted from event files in a circular area of radius 8 arcmin. Background subtraction was performed linking each data file to a standard background file (the latest available at $\mathrm{ASDC}^{1}$ in November 1999). ASDC standard response matrices were also used. In our analysis we used data in the $0.4-4.0 \mathrm{keV}$ energy band.

A larger extraction radius of 13 arcmin was used for MECS. Background subtraction was performed using a $1120 \mathrm{ks}$

\footnotetext{
${ }^{1}$ Calibration files repository: ftp.asdc.asi.it/pub/sax/cal/.
} 
Table 1. Journal of BeppoSAX observations.

\begin{tabular}{llllllllll}
\hline \hline Number & $\begin{array}{l}\text { Start epoch } \\
\text { (UT) }\end{array}$ & $\begin{array}{l}\text { LECS } \\
\text { exposure }\end{array}$ & $\begin{array}{l}\text { LECS } \\
\text { count rate }\end{array}$ & $\begin{array}{l}\text { MECS3 } \\
\text { exposure }\end{array}$ & $\begin{array}{l}\text { MECS3 } \\
\text { count rate }\end{array}$ & $\begin{array}{l}\text { HPGSPC } \\
\text { exposure }\end{array}$ & $\begin{array}{l}\text { HPGSPC } \\
\text { count rate }\end{array}$ & $\begin{array}{l}\text { PDS } \\
\text { exposure }\end{array}$ & $\begin{array}{l}\text { PDS } \\
\text { count rate }\end{array}$ \\
\hline 1 & 17 Mar. 07:08 & $4888 \mathrm{~s}$ & $6.22 \pm 0.04$ & $10108 \mathrm{~s}$ & $6.92 \pm 0.03$ & $4262 \mathrm{~s}$ & $21.5 \pm 0.3$ & $4407+4273 \mathrm{~s}$ & $18.6 \pm 0.1$ \\
2 & 06 Apr. 10:13 & $7302 \mathrm{~s}$ & $5.83 \pm 0.03$ & $12286 \mathrm{~s}$ & $6.10 \pm 0.02$ & $5628 \mathrm{~s}$ & $18.8 \pm 0.2$ & $5631+5626 \mathrm{~s}$ & $16.8 \pm 0.1$ \\
3 & 06 Sep. 05:51 & $5947 \mathrm{~s}$ & $3.96 \pm 0.03$ & $16858 \mathrm{~s}$ & $4.72 \pm 0.02$ & $7432 \mathrm{~s}$ & $15.7 \pm 0.2$ & $7976+7906 \mathrm{~s}$ & $13.9 \pm 0.1$ \\
4 & 01 Oct. 17:36 & $5079 \mathrm{~s}$ & $9.01 \pm 0.04$ & $25046 \mathrm{~s}$ & $9.81 \pm 0.02$ & $11006 \mathrm{~s}$ & $27.6 \pm 0.1$ & $10897+11018 \mathrm{~s}$ & $22.0 \pm 0.1$ \\
\hline
\end{tabular}

${ }^{a}$ Count rates measured in the energy bands used for the fits, namely: 0.4-4.0 keV (LECS); 4.0-10.0 keV (MECS1); 1.8-10.0 keV (MECS2 and MECS3); 7.0-70.0 keV (HPGSPC); 20.0-140.0 keV (PDS).

${ }^{b}$ For MECS we indicate in the table the count rate for unit MECS3. Unit MECS2 has a similar count rate, while unit MECS1 (where present, i.e. in obs $1 \& 2$ ) has a slightly lower count rate due to thicker ion shield.

${ }^{c}$ For the PDS instrument we quote the exposure time as the sum of the two instrument halves while the count rate is the net count rate in the 4 units of which 2 look at the source at any one time for the quoted exposure times.

background file derived from blank field pointings taken during the satellite Performance Verification phase. Response matrices were generated with XAS according to the source location in the field of view. In our analysis we used data in the 4.0$10.0 \mathrm{keV}$ energy band for MECS1, in the 1.8-10.0 keV energy band for MECS2 and MECS3.

HPGSPC background subtraction was performed using data taken during our observations (but not simultaneously) with the rocking collimator offset in the negative direction. ASDC standard response matrices were used. In our analysis we used data in the 7.0-70.0 keV energy band.

PDS background subtraction used simultaneous data taken with the rocking collimators in offset position (Frontera et al. 1997). In consideration of the source strength the threshold for spurious spike filtering has been $32 \mathrm{cts} / \mathrm{s}$ (instead of the customary $25 \mathrm{cts} / \mathrm{s}$ ), and the PSA correction was not applied. Response matrices were computed with XAS from the configuration recorded in the spectra. In our analysis we used data in the 20.0-140.0 keV energy band.

Given the brightness of the source, the systematics in the calibrations of the instrument play an important role when matching spectra taken with different instruments. This is customarily handled using the standard response matrices and introducing cross-normalisation constants in the spectral fit. Unit MECS3 was used as reference (constant fixed to unity). Obtained cross-normalisations for the other instruments are in good agreement with values widely reported in the literature and recommended by the BeppoSAX team.

\section{Data analysis}

Spectral fits were performed with the software package XSPEC v. 10.0. In the remainder of the paper we refer to all additive or multiplicative spectral components, as well as their parameters, with the mnemonic used in the XSPEC handbook.

Preliminary fits were made with the model WABS (DISKBB + POWERLAW), to account for the direct emission from the two main structures around a black hole accreting matter from a companion in a binary system: the optically thick, geometrically thin, accretion disk (DISKBB, Makishima et al. 1986) and an optically thin Comptonising

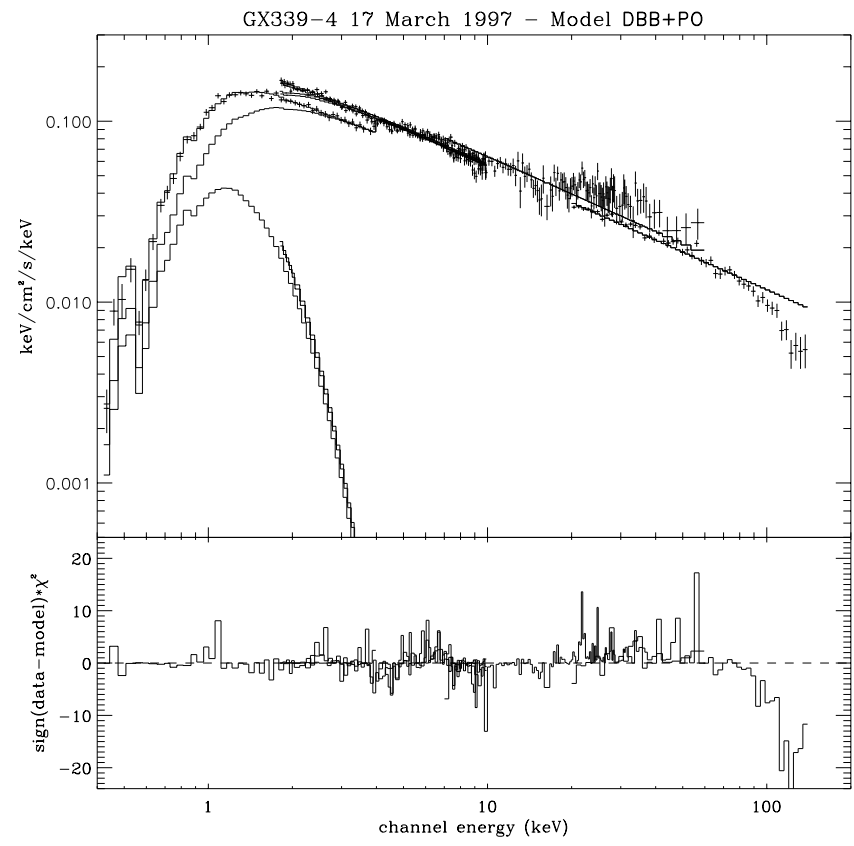

Fig. 2. Result of the WABS (DISKBB + POWERLAW) fit to the spectrum of Mar. 17: unfolded spectrum in top panel, contribution to $\chi^{2}$ in bottom panel.

corona (POWERLAW). The component WABS describes photoelectric absorption due to the interstellar matter (Morrison \& McCammon 1983; Anders \& Ebihara 1982).

Values of the $\chi^{2}$ (see Table 2) indicate that such a simple model does not describe satisfactorily the observed spectra, and Fig. 2 (bottom panel) shows that the main deviations from the model are in the high energy tail of the spectra: the model underestimates the observed spectra at energies around 30-60 keV, and overestimates the spectra at higher energies.

A first possible interpretation of such deviations would be that the spectra have a high energy cutoff, which has not been taken into account in such a simple model: the underestimate at medium energies could be interpreted as the attempt to compensate the lack of a cutoff in the model. A less important deviation is seen at energies around the fluorescence Fe line. 
Table 2. Spectral fits with the WABS (DISKBB + POWERLAW) model.

\begin{tabular}{lllllll}
\hline \hline Component & Parameter & Unit & 17 March & 6 April & 6 September & 1 October \\
\hline WABS & $N_{\mathrm{H}}$ & $10^{21} \mathrm{~cm}^{-2}$ & $5.00_{-0.34}^{+0.36}$ & $5.08_{-0.22}^{+0.28}$ & $4.63_{-0.30}^{+0.35}$ & $6.04_{-0.24}^{+0.25}$ \\
DISKBB & $T_{\text {in }}$ & $\mathrm{keV}$ & $0.301_{-0.022}^{+0.025}$ & $0.351_{-0.026}^{+0.030}$ & $0.360_{-0.040}^{+0.051}$ & $0.363_{-0.018}^{+0.010}$ \\
DISKBB & $K_{\mathrm{DBB}}$ & $(\mathrm{km} / 10 \mathrm{kpc})^{2}$ & $327 ._{-1489}^{+2466}$ & $1147 ._{-468}^{+768}$ & $501_{-290}^{+613}$ & $17866_{-580 .}^{+791 .}$ \\
POWERLAW & $\Gamma$ & & $1.691_{-0.013}^{+0.013}$ & $1.695_{-0.012}^{+0.013}$ & $1.666_{-0.013}^{+0.013}$ & $1.773_{-0.007}^{+0.007}$ \\
POWERLAW & $K_{\mathrm{PO}}$ & photons $/ \mathrm{cm}^{2} \mathrm{~s} \mathrm{keV}($ at $1 \mathrm{keV})$ & $0.286_{-0.007}^{+0.007}$ & $0.252_{-0.006}^{+0.006}$ & $0.188_{-0.004}^{+0.004}$ & $0.454_{-0.007}^{+0.006}$ \\
\hline & $\chi^{2}$ & & 706.78 & 791.97 & 685.33 & 2227.68 \\
& $\chi^{2} /$ d.o.f. & & 1.78 & 1.97 & 1.94 & 5.64 \\
\hline
\end{tabular}

${ }^{a}$ Errors quoted are at the $90 \%$ confidence level.

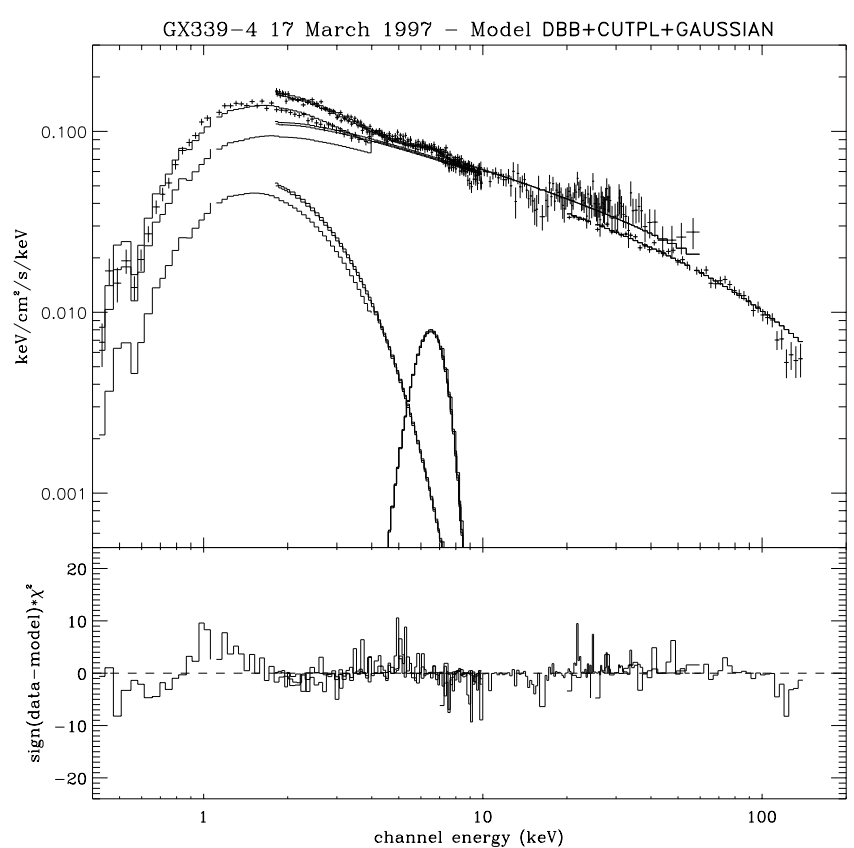

Fig. 3. Result of the WABS (DISKBB + CUTOFFPL + GAUSSIAN) fit to the spectrum of Mar. 17: unfolded spectrum in top panel, contribution to $\chi^{2}$ in bottom panel.

Therefore, a new series of fits were performed with the model WABS (DISKBB + CUTOFFPL + GAUSSIAN): the component CUTOFFPL was introduced to account for the high energy cutoff in the hard tail of the spectra and the component GAUSSIAN for the profile of the Fe fluorescence line. The Fe line energy was kept fixed to the value of $6.4 \mathrm{keV}$ (see Table 3). $\chi^{2}$ values improved for all observations, but they were still too high to indicate good fits. Figure 3 (bottom panel) shows the presence of non negligible modulations, indicating that this improved model cannot describe satisfactorily the shape of the observed spectrum. Figure 3 (top panel) shows clearly that data remain above the model curve in the range about $20-50 \mathrm{keV}$ and are definitely below the model in the hard tail.

The behaviour at energies above $30 \mathrm{keV}$ is suggestive of the presence of a reflected component in the spectra. To test this

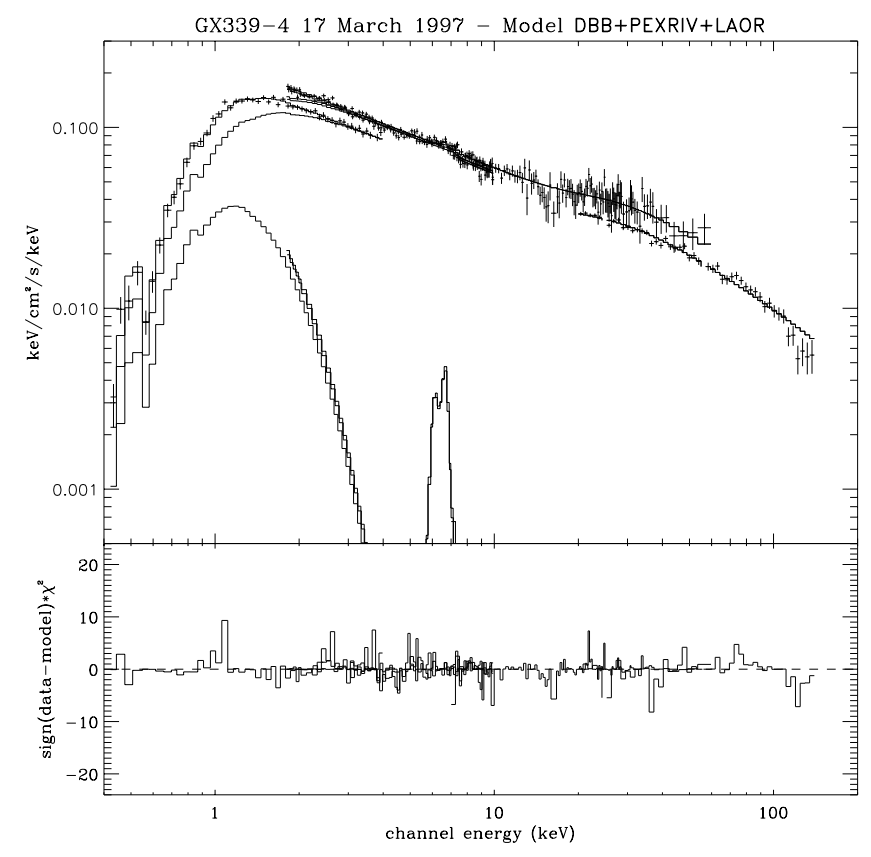

Fig. 4. Result of the WABS (DISKBB + PEXRIV + LAOR) fit to the spectrum of Mar. 17: unfolded spectrum in top panel, contribution to $\chi^{2}$ in bottom panel.

scenario, we introduced the additive model PEXRIV (Magdziarz \& Zdziarski 1995), which describes the Compton effect on a possibly ionized disk, when the incident spectrum is well described by a power law with a high energy cutoff. In conjunction with the PEXRIV component, we performed fits representing the $\mathrm{Fe}$ fluorescence line with the component LAOR (Laor 1991), which describes the line profile from an accretion disk.

Fitting results (see Table 4) indicate clearly that the Compton reflection scenario gives a good description of our data, and Fig. 4 shows how good is the agreement of the assumed model with the data.

\section{Discussion}

Our results clearly show that the source was in the hardlow state during all four observations (see Table 4 for numerical results and Fig. 4 for the unfolded spectrum). Count 
Table 3. Spectral fits with the WABS (DISKBB + CUTOFFPL + GAUSSIAN) model.

\begin{tabular}{lllllll}
\hline \hline Component & Parameter & Unit & 17 March & 6 April & 6 September & 1 October \\
\hline WABS & $N_{\mathrm{H}}$ & $10^{21} \mathrm{~cm}^{-2}$ & $3.18_{-0.29}^{+0.70}$ & $3.83_{-0.25}^{+0.26}$ & $2.88_{-0.17}^{+0.18}$ & $3.73_{-0.12}^{+0.13}$ \\
DISKBB & $T_{\text {in }}$ & $\mathrm{keV}$ & $0.757_{-0.240}^{+0.096}$ & $0.664_{-0.064}^{+0.075}$ & $0.937_{-0.047}^{+0.043}$ & $0.877_{-0.022}^{+0.021}$ \\
DISKBB & $K_{\text {DBB }}$ & $(\mathrm{km} / 10 \mathrm{kpc})^{2}$ & $50.24_{-13.77}^{+120.44}$ & $81.2_{-24.6}^{+41.0}$ & $19.4_{-2.6}^{+3.3}$ & $72.0_{-5.8}^{+6.6}$ \\
CUTOFFPL & $\Gamma$ & & $1.446_{-0.082}^{+0.127}$ & $1.463_{-0.054}^{+0.045}$ & $1.283_{-0.053}^{+0.052}$ & $1.262_{-0.031}^{+0.030}$ \\
CUTOFFPL & $E_{\text {cut }}$ & $\mathrm{keV}$ & $150.7_{-31.1}^{+91.2}$ & $147.9_{-22.5}^{+27.3}$ & $105.5_{-11.9}^{+14.9}$ & $74.5_{-3.8}^{+4.2}$ \\
CUTOFFPL & $K_{\text {cutpl }}$ & photons $/ \mathrm{cm}^{2} \mathrm{~s} \mathrm{keV}(\mathrm{at} 1 \mathrm{keV})$ & $1.77_{-0.30}^{+0.56}$ & $1.65_{-0.19}^{+0.16}$ & $0.85_{-0.01}^{+0.01}$ & $1.59_{-0.11}^{+0.12}$ \\
GAUSSIAN & $\sigma$ & $\mathrm{keV}$ & $0.84_{-0.41}^{+0.13}$ & $0.65_{-0.19}^{+0.24}$ & $0.95_{-0.17}^{+0.17}$ & $1.07_{-0.09}^{+0.09}$ \\
GAUSSIAN & $K_{\text {line }}$ & photons cm ${ }^{-2} \mathrm{~s}^{-1}\left(\times 10^{-3}\right)$ & $2.63_{-1.68}^{+1.57}$ & $1.42_{-0.54}^{+0.81}$ & $2.56_{-0.65}^{+0.76}$ & $7.61_{-0.95}^{+1.01}$ \\
\hline & $\chi^{2}$ & & 528.90 & 546.66 & 429.43 & 698.87 \\
& $\chi^{2} /$ d.o.f. & & 1.37 & 1.37 & 1.22 & 1.79 \\
\hline
\end{tabular}

${ }^{a}$ The Fe-line energy $E_{\text {line }}$ in the component GAUSSIAN has been fixed to $6.4 \mathrm{keV}$.

${ }^{b}$ Errors quoted are at the $90 \%$ confidence level.

Table 4. Spectral fits with the WABS (DISKBB + PEXRIV + LAOR) model.

\begin{tabular}{|c|c|c|c|c|c|c|}
\hline Component & Parameter & Unit & 17 March & 6 April & 6 September & 1 October \\
\hline WABS & $N_{\mathrm{H}}$ & $10^{21} \mathrm{~cm}^{-2}$ & $4.76_{-0.35}^{+0.31}$ & $4.66_{-0.22}^{+0.14}$ & $4.46_{-0.16}^{+0.06}$ & $5.39_{-0.13}^{+0.14}$ \\
\hline DISKBB & $T_{\text {in }}$ & $\mathrm{keV}$ & $0.32_{-0.04}^{+0.05}$ & $0.43_{-0.04}^{+0.04}$ & $0.41_{-0.03}^{+0.04}$ & $0.49_{-0.04}^{+0.02}$ \\
\hline DISKBB & $K_{\mathrm{DBB}}$ & $(\mathrm{km} / 10 \mathrm{kpc})^{2}$ & $1847 ._{-1033}^{+2545}$ & $378.6_{-153.5}^{+274.1}$ & $179.7_{-40.9}^{+104.7}$ & $397.4_{-43.3}^{+67.7}$ \\
\hline PEXRIV & $\Gamma$ & & $1.73_{-0.03}^{+0.04}$ & $1.67_{-0.03}^{+0.04}$ & $1.72_{-0.04}^{+0.01}$ & $1.75_{-0.01}^{+0.01}$ \\
\hline PEXRIV & $E_{\text {cut }}$ & $\mathrm{keV}$ & $533.8_{-161.0}^{+461.8}$ & $285.5_{-53.6}^{+101.9}$ & $802.7_{-152.6}^{+468.5}$ & $264.8_{-18.5}^{+21.6}$ \\
\hline PEXRIV & Rel.Refl & $\Omega / 2 \pi$ & $0.80_{-0.13}^{+0.08}$ & $0.70_{-0.10}^{+0.12}$ & $0.88_{-0.11}^{+0.03}$ & $0.90_{-0.04}^{+0.04}$ \\
\hline PEXRIV & $\mathrm{Fe}_{\text {abund }}$ & Relative to solar abundance & $3.68_{-1.05}^{+0.71}$ & $4.85_{-1.32}^{+1.51}$ & $2.85_{-0.46}^{+0.93}$ & $3.50_{-0.53}^{+0.47}$ \\
\hline PEXRIV & $x_{\mathrm{i}}$ & & $\begin{array}{l}0.37 \\
(<16.29)\end{array}$ & $\begin{array}{l}4.73 \times 10^{-2} \\
(<15.31)\end{array}$ & $\begin{array}{l}5.55 \times 10^{-12} \\
(<0.07)\end{array}$ & $\begin{array}{l}9.12 \times 10^{-6} \\
(<36.12)\end{array}$ \\
\hline PEXRIV & $K_{\text {Pexriv }}$ & photons $/ \mathrm{cm}^{2} \mathrm{~s} \mathrm{keV}$ (at $1 \mathrm{keV}$ ) & $0.293_{-0.013}^{+0.014}$ & $0.237_{-0.006}^{+0.013}$ & $0.195_{-0.006}^{+0.003}$ & $0.416_{-0.017}^{+0.009}$ \\
\hline \multirow[t]{5}{*}{ LAOR } & $K_{\text {Laor }}$ & photons $\mathrm{cm}^{-2} \mathrm{~s}^{-1}\left(\times 10^{-4}\right)$ & $8.33_{-6.20}^{+3.55}$ & $5.13_{-2.03}^{+2.84}$ & $5.33_{-2.23}^{+2.64}$ & $16.90_{-12.22}^{+3.24}$ \\
\hline & Flux & $\operatorname{erg~cm}{ }^{-2} \mathrm{~s}^{-1}\left(\times 10^{-9}\right)$ & 5.98 & 5.21 & 4.34 & 7.46 \\
\hline & Luminosity & $\operatorname{erg~s}^{-1}\left(\times 10^{37}\right)$ & 2.24 & 1.95 & 1.63 & 2.79 \\
\hline & $\chi^{2}$ & & 391.86 & 409.86 & 340.87 & 446.73 \\
\hline & $\chi^{2} /$ d.o.f. & & 1.02 & 1.04 & 0.99 & 1.17 \\
\hline \multicolumn{7}{|c|}{ Cross-normalisation constants with reference to MECS3 = unity } \\
\hline & LECS & & 0.8145 & 0.8065 & 0.7921 & 0.8139 \\
\hline & MECS1 & & 0.9768 & 0.9758 & - & - \\
\hline & MECS2 & & 0.9723 & 0.9731 & 0.9806 & 0.9743 \\
\hline & HPGSPC & & 1.056 & 1.044 & 1.048 & 1.019 \\
\hline & PDS & & 0.8116 & 0.8492 & 0.8096 & 0.7834 \\
\hline
\end{tabular}

a The following parameters have been fixed: Fe-line energy $E_{\text {Laor }}$ in the component LAOR to $6.4 \mathrm{keV}$; the disk inclination angle, present in both components PEXRIV and LAOR, to the value $\cos i=0.5$.

${ }^{b}$ Errors quoted are at the $90 \%$ confidence level.

$c$ Reported fluxes and luminosities are relative to the 0.4-140 kev energy band. Luminosities assume a distance of 5.6 kpc. 
rates and luminosities for each observation are all within a factor of two from each other (see Tables 1 and 4) and are typical of GX 339-4 in its hard-low state (Zdziarski et al. 1998; Ilovaisky et al. 1986; Mendez \& van der Klis 1997; Belloni et al. 1999; Wilms et al. 1999). The spectra are also resemblant of black hole candidates in such spectral state, being mainly dominated by a power law, with a weak soft excess at lower energies (around $1 \mathrm{keV}$ ). The spectral indices of such power laws are also typical of hard-low states, and confirm that the spectra are generated in an optically thin region, assuming they are due to Comptonisation. The large solid angle required by the reflection component suggests the presence of an extended accretion disk, despite the fact that the direct emission from it accounts only for a small fraction of the soft flux (about 3-6\% in the $0.4-10 \mathrm{keV}$ band).

Very little we can argue from the ionisation parameter: all confidence intervals are consistent with zero, but at the same time have very high upper limits. Equivalent widths of the Fe line (from Tables 3 and 4) are also consistent with previous value in litterature (Ueda et al. 1994; Zdziarski et al. 1998; Dolan et al. 1987), lying in the range 50-106 eV.

The values of the high energy cutoff of the incident spectrum require some comments. Such values are clearly outside of the energy range of BeppoSAX instruments, but their determination is needed, in order to constrain accurately the reflected spectrum. They seem not unreasonable. If the cutoff energy were inside the BeppoSAX energy range, the incident spectrum at higher energies would not provide enough photons to generate such an intense reflected component. The absence of a cutoff below $140 \mathrm{keV}$ agrees with previous results (Dolan et al. 1987; Bouchet et al. 1993). In order to confirm the presence of a high energy cutoff in the near $\mathrm{MeV}$ range further investigations, e.g. with Integral, are required.

Acknowledgements. The BeppoSAX satellite has been funded and operated by the Italian Space Agency (ASI). The Rossi XTE ASM data have been retrieved from MIT RXTE web site.

\section{References}

Anders, E., \& Ebihara, M. 1982, GeCoA, 46, 2363

Belloni, T., Méndez, M., van der Klis, M., Lewin, W. H. G., \& Dieters, S. 1999, ApJ, 519, L159
Belloni, T., et al., 2003 in New Views on Microquasars, the fourth Microquasar Workshop, ed. Ph. Durouchoux, Y. Fuchs, \& J. Rodriguez (India: Published by the Center for space Physics), 75 Boella, G., Butler, R. C., Perola, G. C., et al. 1997, A\&AS, 122, 299

Bouchet, L., Jourdain, E., Mandrou, P., et al. 1993, ApJ, 407, 739

Chiappetti, L., \& Dal Fiume, D. 1997, In 5th International Workshop on Data Analysis in Astronomy, ed. V. Di Gesù, M. J. B. Duff, A. Heck, et al., 101

Chiappetti, L., Haardt, F., Treves, A., et al. 1998, In The Active X-ray Sky: Results from BeppoSAX and RXTE, Nucl. Phys. B (Proc. Suppl.), 69(1-3), 340

Chiappetti, L., Maraschi, L., Tavecchio, F., et al. 1999, ApJ, 521, 552

Corbel, S., Nowak, M. A., Fender, R. P., et al. 2003, A\&AS, 400, 1007

Dolan, J. F., Crannell, C. J., Dennis, B. R., \& Orwig, L. E. 1987, ApJ, 322,324

Frontera, F., Costa, E., Dal Fiume, D., et al. 1997, A\&AS, 122, 357

Homan, J., Wijnands, R., van der Klis, M., et al. 2001, ApJS, 132, 377

Ilovaisky, S. A., Chevalier, C., Motch, C., \& Chiappetti, L. 1986, A\&AS, 164, 67

Kong, A. K. H., Kuulkers, E., Charles, P. A., \& Homer, L. 2000, MNRAS, 312, L49

Laor, A. 1991, ApJ, 376, 90

Magdziarz, P., \& Zdziarski, A. A. 1995, MNRAS, 273, 837

Makishima, K., Maejima, Y., Mitsuda, K., et al. 1986, ApJ, 308, 635

Markert, T. H., Clark, G. W., Lewin, W. H. G., Schnopper, H. W., \& Sprott, G. F. 1973a, IAU Circ. 2483

Markert, T. H., Canizares, C. R., Clark, G. W., et al. 1973b, ApJ, 184, L67

Markoff, S., Nowak, M., Corbel., S., et al. 2003, A\&AS, 397, 645

Mendez, M., \& van der Klis, M. 1997, ApJ, 479, 926

Morrison, R., \& McCammon, D. 1983, ApJ, 270, 119

Nowak, M. A., Wilms, J., \& Dove, J. B. 2002, MNRAS, 332, 856

Revnivtsev, M., Gilfanov, M., \& Churazov, E. 2001, A\&AS, 380, 520

Samimi, J., Share, G. H., Wood, K., et al. 1979, Nature, 278, 434

Smith, D. M., Belloni, T., Heindl, W. A., et al. 2002, IAU circ. 7912

Tanaka, Y., \& Lewin, W. H. G. 1995 in X-ray Binaries, ed. W. H. G. Lewin, J. van Paradijs, \& E. P. J. van den Heuvel (Cambridge: Univ. Press), 126

Ueda, Y., Ebisawa, K., \& Done, C. 1994, PASJ, 46, 107

Wardziński, G., Zdziarski, A. A., Gierliński, M., et al. 2002, MNRAS, 337,829

Wilms, J., Nowak, M. A., Dove, J. B., Fender, R. P., \& di Matteo, T. 1999, ApJ, 522, 460

Zdziarski, A. A., Poutanen, J., Mikolajewska, J., et al. 1998, MNRAS, 301,435 